\title{
Correspondence
}

\section{EVOKED POTENTIAL EVIDENCE OF CRANIAL NERVE AND CNS INVOLVEMENT IN CHRONIC RELAPSING POLYRADICULONEUROPATHY}

\section{To the Editor:}

Evidence of CNS demyelination has been recently provided with magnetic resonance imaging in patients with chronic inflammatory demyelinating polyradiculoneuropathy (CRP). ${ }^{1-3}$ On the other hand, there are reports giving clinical, electrophysiological and pathlogical evidence of combined central and peripheral myelinopathy in patients with multiple sclerosis (MS). ${ }^{3}$ Findings on visual and brainstem auditory evoked potentials (VEPs and BAEPs) suggest CNS and auditory nerve involvement in CRP.I We recorded VEPs and BAEPs in six patients with CRP to provide further evidence of the subclinical lesions of the 2nd and 8th cranial nerves, and of CNS demyelination. We also attempted to establish possible correlations between EP alterations and the clinical stage of the disease by carrying out serial EP recordings in some patients. On clinical examination, none of the patients was hypoacusic. Cranial nerves were affected in two patients. In one, bilaterally pale optic disks were noted, in another facial diplegia was present. Two patients demonstrated a rapid low amplitude distal tremor of the upper limbs. In 3 patients nonspecific alterations of EEG were also documented (unilateral or bilateral slowing of background activity).

In summary, the following BAEPs findings were observed: interpeak interval I-II was altered in 7 ears of 4 patients; in 3 ears of 3 of these patients an altered I-III interpeak interval was also present; interpeak interval III-V was altered in 5 ears of 4 patients; interpeak interval I-V was altered in 3 ears of 3 patients and was markedly asymmetric during 3 recording sessions; in 3 ears of 3 patients - recorded during a period of clinical worsening - the alterations were characterized by an almost totally unstructured reponse (identification of different waves became impossible); one of these patients recovered the most important BAEP components (Waves 1, 111, and V) during the next recording session at the time of clinical improvement. VEPs provided evidence of a delayed PIO0 wave in 7 eyes in 4 patients (one of them presenting with bilateral optic disk pallor). These findings suggested the presence of a lesion along the visual pathways.

Our results confirm the involvement of cranial nerves in CRP. In fact, BAEP abnormalities of interpeak intervals I-II, IIII, I-V could be due to disorders of the auditory nerve or of the pons. The relevance of VEP findings is emphasized by the fact that in 6 of 7 affected eyes the alteration of VEPS was confirmed at different spatial frequencies of the stimulating checkerboard and in subsequent recording sessions. In the largest series of patients affected by CRP, the frequency of cranial nerve involvement ranged between 20 and $50 \%$. The audi- tory nerve has never been reported to be affected and optic atrophy has been demonstrated only in one patient. ${ }^{4}$

In addition, our findings provide further evidence of CNS demyelination in CRP. In fact, VEP alterations, even if not discriminatory between optic nerve and retrochiasmatic lesions, are indicative of central demyelination, in that optic nerve fibers are involved. Moreover, even if I-II, I-III and I-V interpeak intervals are not associated with certainty to specific CNS lesions, the presence of prolonged III-V interpeak intervals in 2 patients (2 ears) and the total disruption of the BAEPs in 3 patients ( 3 ears) strongly suggest the possibility of brainstem demyelinating lesions in CRP. These considerations are also indirectly supported by the presence of nonspecific EEG alterations in 3 patients.

The hypothesis of a correlation between the severity of EPS alterations and the clinical course was not demonstrated by our results. In fact, our BAEP findings showed a certain variability independent of the improving or worsening phase of the disease. VEP alterations instead appeared to be permanent throughout the different recording sessions.

These observations support the existence of a central-pcripheral inflammatory demyelinating syndrome. $1-3$ Whether this indicates the existence of a continuum between MS and CRP or is the manifestation of a separate pathogenetic entity with combined demyelination will require further studies.

$$
\begin{array}{rr}
\text { Gian Luigi Gigli, } & \text { Augusto Carlesimo, } \\
\text { Salvatore Mazza, } & \text { Girolamo Di Trapami, } \\
\text { Regulo Hernandez, } & \text { Pietro Tomassetti, } \\
\text { and Università Cartolica del S. Cuore, } \\
\text { Rome, Italy, }
\end{array}
$$

1. Thomas PK, Walker RWH, Rudge P, Morgan-Huges JA, King RHM, Jacobs JM, Mills KR, Ormerod IEC, Murray NMF McDonald WI. Chronic demyelinating peripheral neuropathy associated with multifocal central nervous system demyelination. Brain 1987; 110:53-76.

2. Mendell JR, Kolkin S, Tissell JT, Weiss KL, Chakeres DW, Rammohan $\mathrm{KW}$. Evidence for central nervous system demyelination in chronic inflammatory demyelinating polyradiculoneuropathy. Neurology 1987; 37: 1291-1294.

3. Rubin, M, Karpati G, Carpenter S. Combined central and peripheral myelinopathy. Neurology 1987; 37: 1287-1290.

4. Dyck PJ, Lois AC, Ohta M, Bastron JA, Okazaki H, Groover RV. Chronic inflammatory polyradiculoneuropathy. Mayo Clin Proc 1975; 50: 621-637. 